MATEC Web of Conferences 41, 05002 (2016)

DOI: $10.1051 /$ matecconf/20164105002

(C) Owned by the authors, published by EDP Sciences, 2016

\title{
Evolutionary construction of Runge-Kutta-Nyström pairs of orders 5(4)
}

\author{
I. Th. Famelis ${ }^{1}$, S. Tsitmidelis ${ }^{2}$ and Ch. Tsitouras ${ }^{3, a}$ \\ ${ }^{1}$ TEI of Athens, Department of Electronics Engineering, Egaleo Campus, GR12210, Greece \\ ${ }^{2}$ TEl of Sterea Hellas, Department of Mechanical Engineering, Psahna Campus, GR34400, Greece \\ ${ }^{3}$ TEl of Sterea Hellas, Department of Automation Engineering, Psahna Campus, GR34400, Greece
}

\begin{abstract}
Runge - Kutta - Nyström pairs of orders 5 and 4 are well suited for the solution of Initial value problem of second order. During the last decades were pushed aside by the pairs of orders 6 and 4 share the same number cost. Namely, 5 stages per step. Here we propose an alternative case of such pairs using only four stages per step. This was achieved by solving the corresponding equations of condition by the technique of Differential Evolution. Finally some numerical tests justify our effort.
\end{abstract}

\section{Introduction}

Explicit Runge - Kutta - Nyström pairs [1-4] are amongst the most popular methods for integrating Initial Value Problems of second order with the special form,

$$
y^{\prime \prime}=f(x, y), y\left(x_{0}\right)=y_{0}, y^{\prime}\left(x_{0}\right)=y_{0}^{\prime} .
$$

They advance the solution from $x_{n}$ to $x_{n+1}=x_{n}+h_{n}$, under the following scheme:

$$
\begin{gathered}
y_{n+1}=y_{n}+h_{n} y_{n}^{\prime}+h_{n}^{2} \sum_{i=1}^{s} b_{i} f_{i}, \\
y_{n+1}^{\prime}=y_{n}^{\prime}+h_{n} \sum_{i=1}^{s} b_{i}^{\prime} f_{i}, \\
f_{i}=f\left(x_{n}+c_{i} h_{n}, y_{n}+c_{i} h_{n} y_{n}^{\prime}+h_{n}^{2} \sum_{j=1}^{i-1} a_{i j} f_{j}\right),
\end{gathered}
$$

where $s$ the number of stages per step and $A \in \mathbb{R}^{s \times s}$, $b^{T}, c \in \mathbb{R}^{s}$ the coefficients of the method. The above formula is equivalent to Taylor series expansion of order $p$. This means

$$
y_{1}-y\left(x_{1}\right)=\mathrm{O}\left(h_{1}^{p+1}\right)
$$

and

$$
y_{1}^{\prime}-y^{\prime}\left(x_{1}\right)=\mathrm{O}\left(h_{1}^{p+1}\right)
$$

\footnotetext{
a Corresponding author: tsitouras@mail.teiste.gr
}

Along with the above formula we may deduce another approximation of order $q<p$ at no cost. Namely

$$
\hat{y}_{n+1}=y_{n}+h_{n} y_{n}^{\prime}+h_{n}^{2} \sum_{i=1}^{s} \hat{b}_{i} f_{i} .
$$

Then a step size control algorithm is set by

$$
h_{n+1}=0.9 h_{n} \frac{\varepsilon}{\sigma}
$$

with

$$
\sigma=h_{n}^{p-q-1}\left\|y_{n+1}-\hat{y}_{n+1}\right\|,
$$

and $\varepsilon$ a requested tolerance. The formula above is used when $\varepsilon>\sigma$. Otherwise the advance from $x_{n}$ to $x_{n+1}=x_{n}+h_{n}$ is aborted and the new smaller step $\tilde{h}_{n}=h_{n+1}$ is used for advancing from $x_{n}$ to $x_{n+1}=x_{n}+\tilde{h}_{n}$. For details see [5].

\section{The new method}

For the derivation of a pair of orders 5(4) we have to solve various equations of condition. According to standard literature [6], these are given in Table 1.

In order to solve these 24 equations we set $s=5$ and get 18 coefficients

$$
\begin{aligned}
& a_{21}, a_{31}, a_{32}, a_{41}, a_{42}, a_{43}, \\
& b_{1}, b_{2}, b_{3}, b_{4}, \hat{b}_{1}, \hat{b}_{2}, \hat{b}_{3}, \hat{b}_{4}, \\
& c_{1}, c_{2}, c_{3}, c_{4}
\end{aligned}
$$


Table 1. Equations of condition for a RKN 5(4) pair.

$$
\begin{aligned}
& b e=\frac{1}{2} \quad b c=\frac{1}{6} \\
& b A e=\frac{1}{24} \quad b c^{2}=\frac{1}{12} \\
& b A c=\frac{1}{120} \quad b\left(c^{*} A e\right)=\frac{1}{40} \\
& b c^{3}=\frac{1}{20} \quad \hat{b} e=\frac{1}{2} \\
& \hat{b} c=\frac{1}{6} \quad \hat{b} A e=\frac{1}{24} \\
& \hat{b} c^{2}=\frac{1}{12} \quad b^{\prime} c^{4}=\frac{1}{5} \\
& b^{\prime} e=1 \quad b^{\prime} c=\frac{1}{2} \\
& b^{\prime} A e=\frac{1}{6} \quad b^{\prime} c^{2}=\frac{1}{3} \\
& b^{\prime} A c=\frac{1}{24} \quad b^{\prime}\left(c^{*} A e\right)=\frac{1}{8} \\
& b^{\prime} c^{3}=\frac{1}{4} \quad b^{\prime} A^{2} e=\frac{1}{20} \\
& b^{\prime} A c^{2}=\frac{1}{60} \quad b^{\prime}\left(c^{*} A c\right)=\frac{1}{30} \\
& b^{\prime}(A e)^{2}=\frac{1}{20} \quad b^{\prime}\left(c^{2} * A e\right)=\frac{1}{10}
\end{aligned}
$$

We set

$$
a_{5 j}=b_{j}, j=1,2,3,4
$$

and

$$
c_{5}=1
$$

Thus the fifth stage can be used as first stage of the next step (FSAL). Then the cost of the pair is only 4 stages per step. We also set

$$
\hat{b}_{5}=-\frac{1}{16} \neq b_{5}=0
$$

distinguishing lower and higher order formulas.

Bettis [7] published among the first such a pair at a cost of 5 stages per step. Even if the free coefficients are less than equations, Runge - Kutta experience dictates us to proceed.

The technique of Differential Evolution [8] is preferred. We formed the fitness function

$$
\begin{aligned}
g\left(A, c, b, b^{\prime}, \hat{b}\right) & =\left(b e-\frac{1}{2}\right)^{2}+\left(b c-\frac{1}{6}\right)^{2}+ \\
+ & \left(b A e-\frac{1}{24}\right)^{2}+\cdots+ \\
+ & \left(b^{\prime}(A e)^{2}-\frac{1}{20}\right)^{2}+\left(b^{\prime}\left(c^{2} * A e\right)-\frac{1}{10}\right)^{2}
\end{aligned}
$$

from the equations of Table 1. Then we seek solutions setting safety factor $F=0.8$, crossover $C R=0.95$ and maximum iterations to 900 .

We found tenths of solutions with accuracy of 16 digits. Lower accuracy on the coefficients is not accepted since we may experience problems with consistency of the pair. A particular solution is given in Table 2.

Table 2. Coefficients of the new RKN 5(4) pair.

$$
\begin{aligned}
& a_{21}=0.03540262331616879 \\
& a_{31}=0.00334215697175581 \\
& a_{32}=0.01295154567462482 \\
& a_{41}=0.1097809262561679 \\
& a_{42}=0.3603866917982281 \\
& a_{43}=0.2381444712334193 \\
& b_{1}=0.04994112866537466 \\
& b_{2}=0.1391690249402197 \\
& b_{3}=0.1640662567463215 \\
& b_{4}=0.1468235896480842 \\
& \hat{b}_{1}=0.2876949142374448 \\
& \hat{b}_{2}=0.5025805236616177 \\
& \hat{b}_{3}=-0.5005027106263353 \\
& \hat{b}_{4}=0.2727272727272727 \\
& \hat{b}_{5}=-0.0625 \\
& b_{1}^{\prime}=0.04994112866537466 \\
& b_{2}^{\prime}=0.1896274870392451 \\
& b_{3}^{\prime}=0.2002077178727353 \\
& b_{4}^{\prime}=0.09965925350010441 \\
& b_{5}^{\prime}=0.09965925350010441 \\
& c_{2}=0.2660925527562498 \\
& c_{3}=0.1805198196674295 \\
& c_{4}=0.6812094344927655 \\
& c_{5}=1, a_{5 j}=b_{j}, j=1,2,3,4
\end{aligned}
$$




\section{Numerical Tests}

We tested our new method along with the corresponding 5(4) pair of Bettis. We choose the celebrated two body problem,

$$
\begin{aligned}
& y_{1}^{\prime \prime}=-\frac{y_{1}}{{\sqrt{y_{1}^{2}+y_{2}^{2}}}^{3}}, \\
& y_{2}^{\prime \prime}=-\frac{y_{2}}{{\sqrt{y_{1}^{2}+y_{2}^{2}}}^{3}},
\end{aligned}
$$

with

$$
\begin{aligned}
& y_{1}(0)=0.5, y_{2}(0)=0, \\
& y_{1}^{\prime}(0)=0, y_{2}^{\prime}(0)=\sqrt{3},
\end{aligned}
$$

and

$$
0<x<6 \pi
$$

The tolerances we run were

$$
\varepsilon=10^{-3}, 10^{-4}, \cdots, 10^{-11} \text {. }
$$

In Tables 3 and 4 we recorded for each tolerance the function evaluations needed for each pair and the digits of accuracy achieved at the end-point.

Table 3. Results for the new pair.

\begin{tabular}{|c|c|c|}
\hline Tolerance & $\begin{array}{c}\text { function } \\
\text { evaluations }\end{array}$ & $\begin{array}{c}\text { digits of } \\
\text { accuracy }\end{array}$ \\
\hline $10^{-3}$ & 253 & 1.8 \\
\hline $10^{-4}$ & 377 & 2.9 \\
\hline $10^{-5}$ & 525 & 3.9 \\
\hline $10^{-6}$ & 641 & 5.3 \\
\hline $10^{-7}$ & 973 & 6.4 \\
\hline $10^{-8}$ & 1541 & 7.5 \\
\hline $10^{-9}$ & 2437 & 8.5 \\
\hline $10^{-10}$ & 3861 & 9.5 \\
\hline $10^{-11}$ & 6113 & 10.5 \\
\hline
\end{tabular}

Interpreting the results from these tables we see a clear advantage of the new method over the older one.
Table 4. Results for the Bettis pair.

\begin{tabular}{|c|c|c|}
\hline Tolerance & $\begin{array}{c}\text { function } \\
\text { evaluations }\end{array}$ & $\begin{array}{c}\text { digits of } \\
\text { accuracy }\end{array}$ \\
\hline $10^{-3}$ & 281 & 1.0 \\
\hline $10^{-4}$ & 416 & 1.8 \\
\hline $10^{-5}$ & 616 & 2.8 \\
\hline $10^{-6}$ & 806 & 3.5 \\
\hline $10^{-7}$ & 1096 & 4.3 \\
\hline $10^{-8}$ & 1731 & 5.4 \\
\hline $10^{-9}$ & 2741 & 6.6 \\
\hline $10^{-10}$ & 4341 & 8.2 \\
\hline $10^{-11}$ & 6876 & 8.6 \\
\hline
\end{tabular}

\section{Acknowledgments}

This research has been co-financed by the European Union (European Social Fund - ESF) and Greek national funds through the Operational Program "Education and Lifelong Learning" of the National Strategic Reference Framework (NSRF) - Research Funding Program: ARCHIMEDES III. Investing in knowledge society through the European Social Fund (grand no.383779/2).

\section{References}

1. C. Tsitouras, J. Comput. Appl. Math., 95, 167-171 (1998).

2. S. N. Papakostas, C. Tsitouras, SIAM J. Sci. Comput., 21, 727-743 (1999).

3. C. Tsitouras, Cele. Mech., 74, 223-230 (1999).

4. C. Tsitouras, I. T. Famelis, New Astron., 17, 469473 (2012).

5. C. Tsitouras, S. N. Papakostas, SIAM J. Sci. Comput., 20, 2067-2088 (1999).

6. C. Tsitouras, I. T. Famelis, J. Math. Chem., 46, 896912 (2009).

7. D. G. Bettis, Cele. Mech., 8, 229-233 (1973).

8. R. Storn, K. Price, J. Glob. Optim., 11, 341--359 (1997). 